\title{
Design Analysis of High Frequency Linear Transformer with Ārtap Framework
}

\author{
Tamás Orosz ${ }^{1,2^{*}}$, Mariusz Stępień ${ }^{3}$, Peter Poór ${ }^{1}$ \\ 1 Department of Theory of Electrical Engineering, Faculty of Electrical Engineering, University of West Bohemia, 30100 Plzen, \\ 2732/8 Univerzitní, Czech Republic \\ 2 MONTANA Knowledge Management Ltd, 1111 Budapest, Lágymányosi u. 11., Hungary \\ ${ }^{3}$ Department of Power Electronics, Electrical Drives and Robotics, Faculty of Electrical Engineering, Silesian University of \\ Technology, 44-100 Gliwice, 2 B. Krzywoustego, Poland \\ *Corresponding author, e-mail: orosz.tamas@montana.hu
}

Received: 11 November 2020, Accepted: 10 December 2020, Published online: 19 April 2021

\begin{abstract}
This paper presents the design and a design analysis of a coaxial, linear transformer. This is a novel high frequency transformer concept for energy conversion. The examined transformer was designed for $1 \mathrm{MHz}$ nominal frequency. One of the main advantages of the proposed transformer design is its simple winding system. It contains only two coaxial copper tubes, which can be easily manufactured and modeled with high precision. One of the key design tasks is the minimization of the leakage inductance. The inductance of the straight coils depends on the ratio of the height and the diameter of the coil. Therefore, a three-dimensional FEM analysis is sufficient to calculate the optimal length of the linear transformer. The planar 2D model and the 3D model of the transformer are presented in this paper. The accuracy of the 2D and 3D calculation results were compared to each other and to the measurements to show the applicability of the planar 2D models. Moreover, the sensitivity of the losses and the leakage inductance with respect to the winding parameters is presented. The dependencies of the design variables on the performance parameters, such as the power mass density and the leakage inductance of this transformer concept were examined. It was shown that the value of the leakage inductance is a linear function of the ratio of the length and the diameter of the transformer windings.
\end{abstract}

\section{Keywords}

FE analysis, DC-DC conversion, transformer, high frequency, optimization

\section{Introduction}

Performance-per-watt requirements are driving higher energy efficiency and density metrics in power conversion technologies [1]. Due to the recent advances in the power semiconductor technologies the operating frequency of these applications became higher. One of the most common structures of the medium and high frequency solid state transformers is the cascaded H-bridge topology. This can be composed from many stages [2, 3], to reach high-current or high-voltage requirements, resolving the power-semiconductor limitations [2, 4-6].

Magnetic components, i.e. transformers and coils, are the cause that limits integration of power converters into the final applications [7]. Although that the magnetic components can limit the integration, they are still a key technology to improve the performance of power transformers [7]. There is a high demand (from the 1950's [7, 8]) from the industry to find new technologies and transformer layouts, which can increase the performance-per-watt ratios $[1,3,7,9,10]$.

There are many papers, which presents loss prediction and the design of magnetic components [1, 2, 4, 7, 9-21]. Most of the design methodologies are still based on the Dowell formulas, which has certain limitations [1, 9, 11, 22] and most of the conventional converter designs applies sandwich windings to increase the coupling of the transformer [20]. However, this design can limit the coupling of the transformer windings. Even though power losses of the main switch and diode should be taken into account, they can be reduced by the reduction of the leakage inductance of the transformer [20].

This paper investigates a novel winding design, a coaxial linear transformer. This simple winding structure consists of only two coaxial cooper tubes, where the inner winding 
is actively cooled by water. Other advantages are very high coupling coefficient, relatively low leakage inductance and high-power density. These advantages are inherent feature of this transformer design [16-18]. High coupling coefficient and low leakage inductance are the results of windings arrangement, high power density is result of efficient water cooling of transformer windings. These benefits can be fully achieved at frequencies above $100 \mathrm{kHz}[17,18]$.

The main parameters of the proposed design were investigated by 2D and 3D FEM software. The 2D FEM calculations were performed originally by Ansys. For the accurate calculation of the leakage inductance, a 3D harmonic magnetic field analysis was performed in COMSOL, the optimal value of the leakage inductance was determined with the use of Ārtap framework, which contains tools and integrated optimization algorithms for robust design optimization and executors for different FEM solvers, like COMSOL or Agros Suite [23-26].

\section{The examined linear transformer}

The prototype of the experimental coaxial linear transformer has been fabricated for laboratory testing (Fig. 1). These design calculations were based on the design principles of a spiral coaxial transformer. The main objectives during the design process were to minimize the leakage inductance and increase the energy density of the transformer. To achieve these objectives and to achieve the highest possible coupling between the primary and the secondary winding, the thickness of the main insulation was selected as small as possible. This insulation was made of a $0.5 \mathrm{~mm}$ wide glass fibre. To minimize the winding losses, the thickness of the windings (copper tubes) should be twice the skin

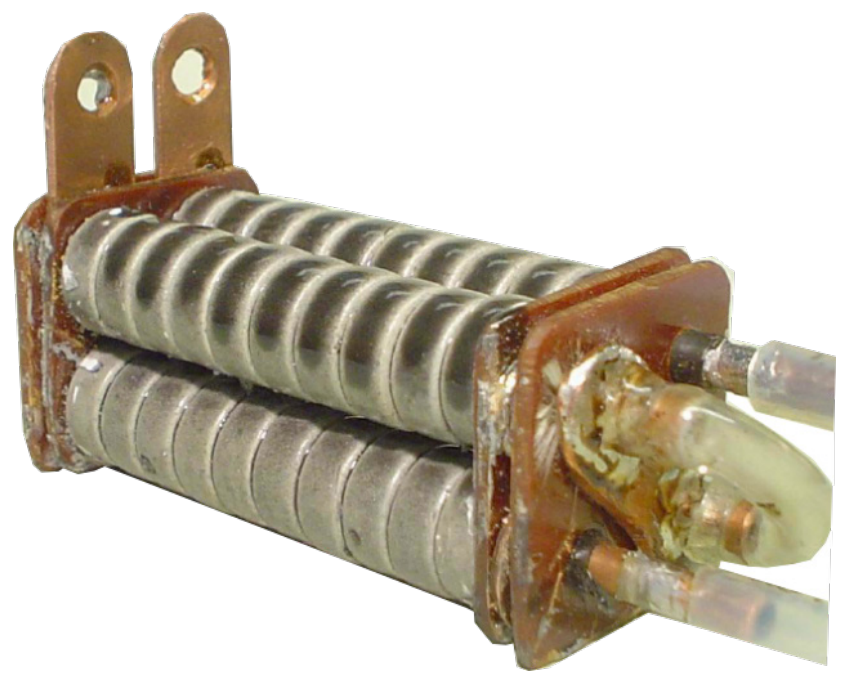

Fig. 1 The examined coaxial linear transformer with $P_{n}=4.3 \mathrm{kVA}$ and 2:1 turn-to-turn ratio depth, which is $67 \mathrm{~m}$ at the nominal working frequency of the transformer $(1 \mathrm{MHz})$. Due to the manufacturing tolerances, this thickness is selected a bit larger, a $0.5 \mathrm{~mm}$ thick copper tubes were selected for our prototype transformer. The cross section of the prototype linear transformer is shown in Fig. 2, the dimensions are summarized in Table 1.

The turn ratio of the designed transformer is $2: 1$, where the nominal voltage in the primary coil is $112.4 \mathrm{~V}$. The nominal currents are $\left(I_{1 n}=39.4 \mathrm{~A}, I_{2 n}=79.8 \mathrm{~A}\right)$ at $P_{n}=4.36 \mathrm{~kW}$ nominal power, with the maximum allowed joule losses $\left(P_{j}\right)$ $20 \mathrm{~W}$. This value was estimated by another FEM calculation $[17,18]$. The transformer power was determined in different working frequencies with and without a ferrite core (Fig. 3). The estimated efficiency of the transformer, with ferrite core at $1 \mathrm{MHz}$ working frequency is $=99.54 \%$. TN13.5/7/5-3F4 type core rings were applied to the design, each of the cores has $5 \mathrm{~mm}$ thickness, so the total height of the core is $48 \mathrm{~mm}$ (Fig. 1), the total length of the transformer
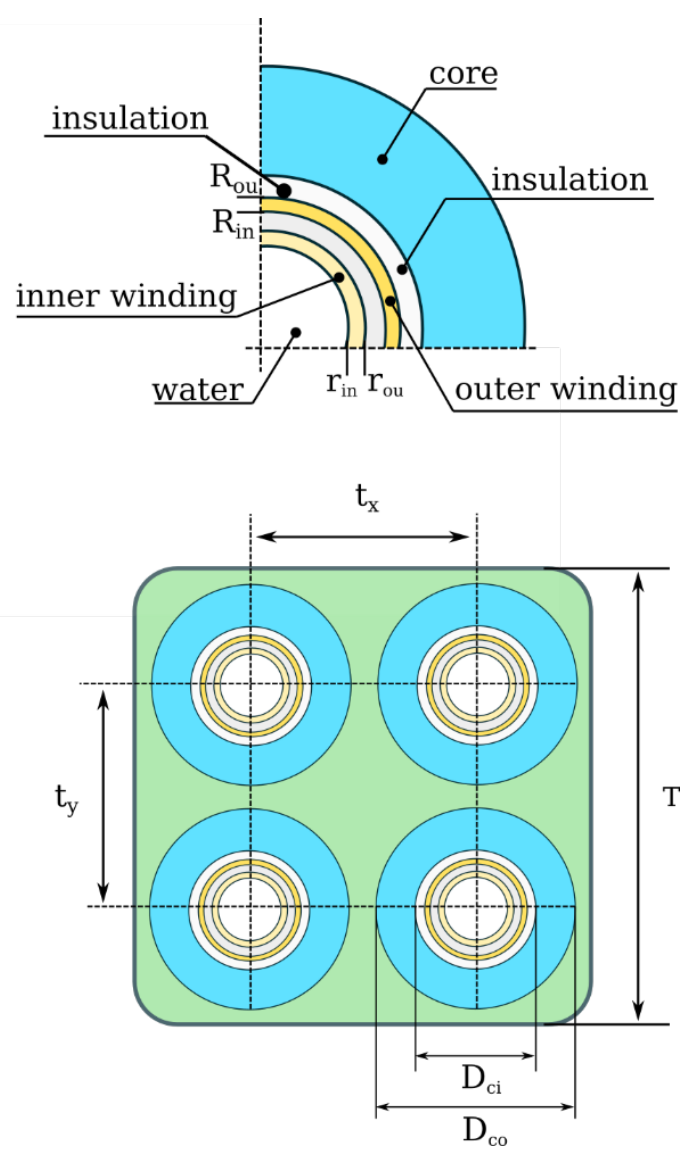

Fig. 2 The examined coaxial linear transformer with $P_{n}=4.3 \mathrm{kVA}$ and 2:1 turn-to-turn ratio

Table 1 The geometrical dimensions of the manufactured transformer

\begin{tabular}{lccccccc}
\hline$r_{i n}$ & $r_{o u}$ & $R_{i n}$ & $R_{o u}$ & $D_{c i}$ & $D_{c o}$ & $t_{x}=t_{y}$ & $T$ \\
\hline$[\mathrm{mm}]$ & {$[\mathrm{mm}]$} & {$[\mathrm{mm}]$} & {$[\mathrm{mm}]$} & {$[\mathrm{mm}]$} & {$[\mathrm{mm}]$} & {$[\mathrm{mm}]$} & {$[\mathrm{mm}]$} \\
1.5 & 2.0 & 2.5 & 3.0 & 7.0 & 13.5 & 15.0 & 30.0 \\
\hline
\end{tabular}




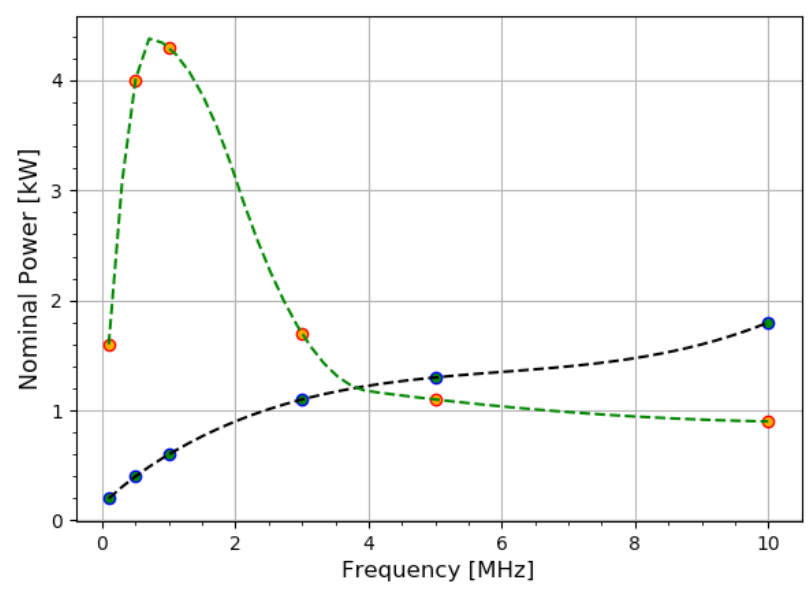

Fig. 3 The nominal power of the proposed linear transformer for different frequencies. The green dashed line represents the nominal power with ferrite core, the blue dashed line represents the air core case

is $60 \mathrm{~mm}$. This distance is equal with the half of the circumference of one turn of the spiral transformer. This selection ensures equivalence between the circular coaxial transformers and the proposed linear transformer [16, 17]. Otherwise, the height/diameter ratio of the applied copper tubes has a significant impact on the leakage impedance of the transformer. This effect is analyzed in next section of paper.

Other important measure of this transformer design is the maximum achievable power density. This value can be determined from the geometrical and electric power data given above. The mass density of the applied copper tubes is $c u=8920 \mathrm{~kg} / \mathrm{m}^{3}$, core $=4700 \mathrm{~kg} / \mathrm{m}^{3}$ for the core and ins $=1200 \mathrm{~kg} / \mathrm{m}^{3}$ for the glass fiber insulation. From these data, the total mass of the linear transformer is $m t=57.1 \mathrm{~g}$, which is the sum of the mass of the copper tubes (14.4 g), the ferrite core $(42.4 \mathrm{~g})$ and the insulation $(0.3 \mathrm{~g})$. In this case, the achievable maximum power density at $1 \mathrm{MHz}$ is $76.3 \mathrm{~kW} / \mathrm{kg}$ with ferrite core, where the output power of the linear transformer is $4.3 \mathrm{~kW}$. The measurements proved $[16,17]$, that this theoretical number can be achieved with the proposed design. However, the performance of the natural water flow based cooling is relatively low, it can be increased significantly with better water cooling.

\section{Design analysis}

The aim of this section is to point out some interesting sensitivity analyses, which can help to understand non-linear behavior of the examined linear coaxial transformer. The subject of the first analyses was to determine the impact of the diameter selection of the inner winding on the losses of the transformer. During the analyses, the acceptable joule losses $\left(P_{j}\right)$ were estimated by finite element analysis, proportionally to the heat dissipation on the surface.
The maximum allowed input voltage $\left(U_{1 N}\right)$ and the allowed maximum $P_{j}$ values are described in Table 2 . The results of this analysis are summarized in Table 1. It can be seen that the nominal power increases linearly with the radius of the inner winding. The presented results are normalized for one winding, the manufactured design contains 4 windings (Fig. 1). In this case, we assumed that the distance of the glass fibre insulation is minimized.

During the second analyses the influence of the core diameter selection on the performance parameters were examined. In this case, the winding dimensions were held constant and the inner diameter of the ferrite core was varied. The maximum value of the magnetic flux density was calculated by a 2D planar FEM analysis.

The inner diameter of the ferrite core $\left(D_{c}\right)$ in the analysis varied from the smallest possible $(6.5 \mathrm{~mm})$ to $16 \mathrm{~mm}$. The minimum core diameter is limited by the maximum allowed value of the magnetic flux density, which guarantees that the core is not saturated. The output maximum possible output power $\left(P_{2 n}\right)$ and the power mass density $(\alpha)$ were examined during the analysis. The analysis was carried out with the following assumptions about the magnetic core: a magnetizing curve is approximated by its relative permittivity $\left(\mu_{\gamma}=2000\right)$ and it is considered as linear till the saturation point, which is $B_{s}=250 \mathrm{mT}$. The hysteresis phenomenon was included only in the core loss calculations. The calculated values are presented in Table 3.

Table 2 The impact of the inner winding diameter selection on the performance parameters on the linear transformer.

\begin{tabular}{lccccc}
\hline $\begin{array}{l}r_{i n} \\
{[\mathrm{~mm}]}\end{array}$ & $\begin{array}{c}P_{j} \\
{[\mathrm{~W}]}\end{array}$ & $\begin{array}{c}\eta \\
{[\%]}\end{array}$ & $\begin{array}{c}P_{2 N} \\
{[\mathrm{~kW}]}\end{array}$ & $\begin{array}{c}\alpha \\
{[\mathrm{kW} / \mathrm{kg}]}\end{array}$ & $\begin{array}{c}U_{1 N} \\
{[\mathrm{~V}]}\end{array}$ \\
\hline$[\mathrm{mm}]$ & {$[\mathrm{mm}]$} & {$[\mathrm{mm}]$} & {$[\mathrm{mm}]$} & {$[\mathrm{mm}]$} & {$[\mathrm{mm}]$} \\
1 & 4 & 99.42 & 0.69 & 45.9 & 48 \\
1.5 & 6 & 99.48 & 1.16 & 59.4 & 50.8 \\
2 & 8 & 99.52 & 1.65 & 68.7 & 53.1 \\
2.5 & 10 & 99.53 & 2.14 & 75.1 & 54.9 \\
3 & 12 & 99.55 & 2.64 & 80 & 56.4 \\
\hline
\end{tabular}

Table 3 The impact of the inner core diameter selection on the performance parameters of the transformer.

\begin{tabular}{lccccc}
\hline $\begin{array}{l}D_{c i} \\
{[\mathrm{~mm}]}\end{array}$ & $\eta$ & $P_{2 N}$ & $\alpha$ & $U_{1 N}$ & $B_{\max }$ \\
{$[\%]$} & {$[\mathrm{kW}]$} & {$[\mathrm{kW} / \mathrm{kg}]$} & {$[\mathrm{V}]$} & {$[\mathrm{mT}]$} \\
\hline$[\mathrm{mm}]$ & {$[\mathrm{mm}]$} & {$[\mathrm{mm}]$} & {$[\mathrm{mm}]$} & {$[\mathrm{mm}]$} & {$[\mathrm{mm}]$} \\
6.4 & 99.23 & 1.29 & 118.6 & 33.4 & 225 \\
8 & 99.39 & 1.62 & 91.1 & 41.4 & 116 \\
10 & 99.53 & 2.14 & 75 & 54.9 & 104 \\
12 & 99.6 & 2.52 & 60.4 & 63.7 & 96 \\
14 & 99.65 & 2.81 & 49.1 & 71.4 & 92 \\
16 & 99.67 & 3.05 & 40.5 & 78.4 & 91 \\
\hline
\end{tabular}




\section{3D sensivity analysis}

Originally, the linear transformer length was selected to be half of the diameter of the reference circular coaxial transformer, to get equivalent magnetic and electrical properties. However, the inductance of the coil heavily depends on the ratio of the coil height and diameter [27].

Inductance calculation is straightforward if the coils are wound with an infinitesimally thin conducting tape with essentially no gaps between turns and when the length of the coil is high, much higher than the diameter of the coil. Technically, this kind of coils does not exist. In our case, the height of the primary coil is $60 \mathrm{~mm}$, which is 10 times higher than the diameter of the outer (secondary) coil.

The leakage inductance of the transformer can be calculated from the magnetic energy:

$W_{m}=\frac{1}{2} L_{s} I^{2}$,

where $W_{m}$ denotes the magnetic energy in $J, L_{s}$ is the leakage inductance in $H$ and $I$ is the current of the reference coil in $A$.

As part of our research, a sweep analysis was performed by Ārtap where the length of the windings was varied from $30 \mathrm{~mm}$ to $100 \mathrm{~mm}$. Ārtap controlled our 3D COMSOL model during the analysis. For the sake of simplicity, the length of the core was always the same as the length of the windings during our calculations. A harmonic magnetic field analysis was performed at $f=1 \mathrm{MHz}$ frequency. The magnetic energy $\left(W_{m}\right)$ and the coil losses $P_{l p}$ in the primary and $P_{s p}$ in the secondary windings were calculated by COMSOL (Fig. 4). The transformer consists of four columns, using this symmetry only one column was modeled by FEM. The total copper losses $\left(P_{c u}\right)$ were calculated by a simple multiplication. The current in the primary and the secondary coils was 38 A and $I_{p}=19$ A-s during the calculation. The previous measurements [16] show that the total copper losses in the prototype are about $2 \mathrm{~W}$, which is $10 \%$ higher than the calculated $1.84 \mathrm{~W}$.

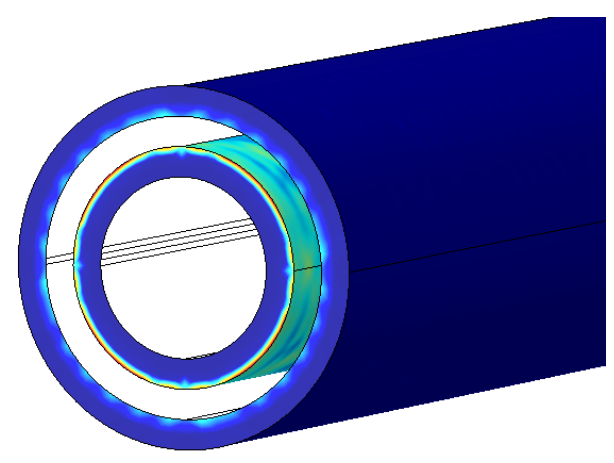

Fig. 4 The influence of the magnetic flux density and the output power on the selection of the core diameter.
The manufacturing tolerances and the 3D mesh can explain this difference. Due to high frequency $(1 \mathrm{MHz})$, the penetration depth in the windings is very small (Fig. 5) and the numerical computation of the magnetic field is computationally expensive. The result of the analysis is presented in Table 4. The last column of this Table contains the magnetic energy density $\left(D_{m}\right)$. This is the ratio of the stored magnetic energy $\left(W_{m}\right)$ and the length of the transformer.

The magnetic energy density and the magnetic energy are plotted in Fig. 6. From this figure it can be seen that the magnetic energy has a quadratic dependence on the length of the transformer. This dependence can more non-linear at the nominal power $\left(I_{p}=38\right.$ A) $[16-18]$. In Fig. 6 we can see a deviation from the curve at $45 \mathrm{~mm}$, it was caused by a numerical computation error. It can be also seen that around $50 \mathrm{~mm}$ there is an inflection point in the magnetic energy density curve. Above this point there is a linear dependency of the magnetic energy on the length of the transformer, from this point the 2D FEM provides accurate or even more accurate results, because some $2 \mathrm{D}$ academic

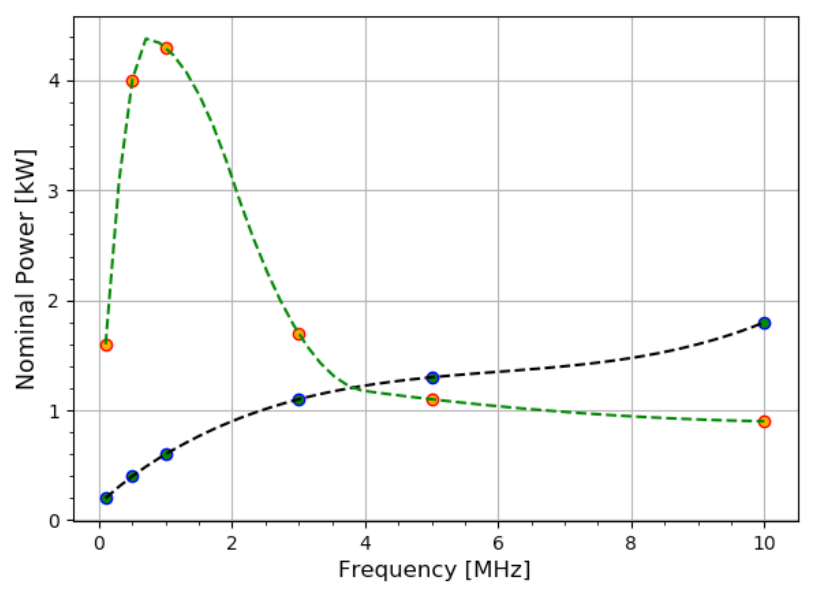

Fig. 5 Power density in the primary and secondary windings.

Table 4 The impact of the winding length on the magnetic energy and the copper losses.

\begin{tabular}{lccccc}
\hline $\begin{array}{l}\text { Lenght } \\
{[\mathrm{mm}]}\end{array}$ & $\begin{array}{c}P_{l p} \\
{[\mathrm{~W}]}\end{array}$ & $\begin{array}{c}P_{l s} \\
{[\mathrm{Ww}]}\end{array}$ & $\begin{array}{c}P_{c u} \\
{[\mathrm{~W}]}\end{array}$ & $\begin{array}{c}W_{m} \\
{[\mathrm{Ww}]}\end{array}$ & $\begin{array}{c}D_{m} \\
{[\mathrm{~W}]}\end{array}$ \\
\hline$[\mathrm{mm}]$ & {$[\mathrm{mm}]$} & {$[\mathrm{mm}]$} & {$[\mathrm{mm}]$} & {$[\mathrm{mm}]$} & {$[\mathrm{mm}]$} \\
0.030 & 0.136 & 0.097 & 0.929 & $2.64 \mathrm{E}-12$ & $8.79 \mathrm{E}-11$ \\
0.035 & 0.156 & 0.108 & 1.058 & $3.07 \mathrm{E}-12$ & $8.77 \mathrm{E}-11$ \\
0.040 & 0.169 & 0.131 & 1.202 & $3.47 \mathrm{E}-12$ & $8.68 \mathrm{E}-11$ \\
0.045 & 0.200 & 0.143 & 1.370 & $4.16 \mathrm{E}-12$ & $9.24 \mathrm{E}-11$ \\
0.050 & 0.191 & 0.154 & 1.381 & $4.32 \mathrm{E}-12$ & $8.63 \mathrm{E}-11$ \\
0.055 & 0.236 & 0.169 & 1.620 & $5.38 \mathrm{E}-12$ & $9.79 \mathrm{E}-11$ \\
0.060 & 0.275 & 0.184 & 1.836 & $6.46 \mathrm{E}-12$ & $1.08 \mathrm{E}-10$ \\
0.070 & 0.318 & 0.215 & 2.132 & $8.24 \mathrm{E}-12$ & $1.18 \mathrm{E}-10$ \\
0.080 & 0.355 & 0.241 & 2.384 & $1.01 \mathrm{E}-11$ & $1.27 \mathrm{E}-10$ \\
0.100 & 0.458 & 0.306 & 3.057 & $1.55 \mathrm{E}-11$ & $1.55 \mathrm{E}-10$ \\
\hline
\end{tabular}



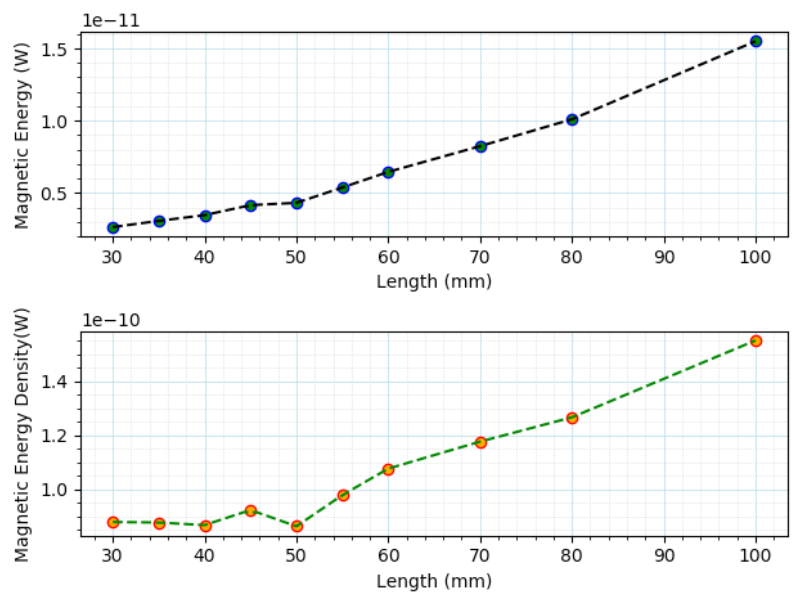

Fig. 6 The dependence of the stored magnetic energy on the length of the linear transformer.

FEM codes provides $h p$-adaptive [24, 25, 28-30] FEM solver, whose higher degree polynomials can better approximate the exponentially decaying magnetic field in the copper tubes. It can be concluded that the optimal length of the transformer core and windings is about $50 \mathrm{~mm}$, which is very close to the manufactured core $(48 \mathrm{~mm})$ and windings (50 and $60 \mathrm{~mm}$ ). A more accurate 3D calculation can consider the end effect, which is caused by the copper tubes

\section{References}

[1] Fu, D., Wang, S. "Novel concepts for high frequency high efficiency transformer design", In: 2011 IEEE Energy Conversion Congress and Exposition, Phoenix, AZ, USA, 2011, pp. 3800-3807. https://doi.org/10.1109/ECCE.2011.6064285

[2] Villar, I., Garcia-Bediaga, A., Viscarret, U., Etxeberria-Otadui, I., Rufer, A. "Proposal and validation of medium-frequency power trans-former design methodology", In: 2011 IEEE Energy Conversion Congress and Exposition, Phoenix, AZ, USA, 2011, pp. 3792-3799.

https://doi.org/10.1109/ECCE.2011.6064284

[3] Shamshuddin, M. A., Rojas, F., Cardenas, R., Pereda, J., Diaz, M., Kennel, R. "Solid State Transformers: Concepts, Classification, and Control", Energies, 13(9), Article number: 2319, 2020. https://doi.org/10.3390/en13092319

[4] Orosz, T., Pánek, D., Karban, P., de Oliveira, R. A. H., Murta Pina, J. "Medium Frequency Transformer Design with Ārtap Framework", In: 2020 27th International Workshop on Electric Drives: MPEI Department of Electric Drives 90th Anniversary (IWED), Moscow, Russia, 2020, pp. 1-4. https://doi.org/10.1109/IWED48848.2020.9069502

[5] Prasai, A., Yim, J.-S., Divan, D., Bendre, A., Sul, S.-K. "A New Architecture for Offshore Wind Farms", IEEE Transactions on Power Electronics, 23(3), pp. 1198-1204, 2008. https://doi.org/10.1109/TPEL.2008.921194

[6] Steiner, M., Reinold, H. "Medium frequency topology in railway applications", In: 2007 European Conference on Power Electronics and Applications, Aalborg, Denmark, 2007, pp. 1-10. https://doi.org/10.1109/EPE.2007.4417570 longer than the core. However, due to the high operating frequency the loss prediction can be calculated more accurately by a $2 \mathrm{D}$ FEM analysis outside this area.

\section{Conclusions}

Power mass density is one of the most important measures to characterize a power transformer design. The coaxial linear transformer is relatively new concept and it provides very high-power mass density. The paper presented a sensitivity analyses and the design considerations of an experimental coaxial linear transformer. The dependencies of the design variables on the performance parameters, such as the power mass density and the leakage inductance of this transformer concept were examined. It was shown that the value of the leakage inductance is a linear function of the ratio of the length and the diameter of the transformer windings. The optimal length of the transformer can be estimated by a 3D FEM calculation and the proposed optimization method in Ārtap framework. The proposed model can be used as a benchmark for the most minute and detail calculations and the selection of the most accurate numerical optimization methodology, according to the no-free lunch theorem of the mathematical optimization.

[7] Magambo, J. S. N. T., Bakri, R., Margueron, X., Le Moigne, P., Mahe, A., Guguen, S, Bensalah, T. "Planar Magnetic Components in More Electric Aircraft: Review of Technology and Key Parameters for DC-DC Power Electronic Converter", IEEE Transactions on Transportation Electrification, 3(4), pp. 831-842, 2017. https://doi.org/10.1109/TTE.2017.2686327

[8] Stephens, D. S. "Lightweight Aircraft Transformers", Transactions of the American Institute of Electrical Engineers, 68(2), pp. 1073-1078, 1949.

https://doi.org/10.1109/T-AIEE.1949.5060052

[9] Bahmani, M. A., Thiringer, T., Kharezy, M. "Design Methodology and Optimization of a Medium-Frequency Transformer for HighPower DC-DC Applications", IEEE Transactions on Industry Applications, 52(5), pp. 4225-4233, 2016. https://doi.org/10.1109/TIA.2016.2582825

[10] Orosz, T. "Evolution and Modern Approaches of the Power Transformer Cost Optimization Methods", Periodica Polytechnica Electrical Engineering and Computer Science, 63(1), pp. 37-50, 2019.

https://doi.org/10.3311/PPee.13000

[11] Dowell, P. L. "Effects of eddy currents in transformer windings", Proceedings of the Institution of Electrical Engineers, 113(8), pp. 1387-1394, 1966. https://doi.org/10.1049/piee.1966.0236

[12] Kvitkovic, J., Pamidi, S. V., Graber, L., Chiocchio, T., Steurer, M., Usoskin, A. "AC Loss and Magnetic Shielding Measurements on 2GHTS Inductive Fault Current Limiter Prototype Modules", IEEE Transactions on Applied Superconductivity, 24(3), pp. 1-4, 2013. https://doi.org/10.1109/TASC.2013.2280845 
[13] Bahmani,M. A., Thiringer, T., Ortega, H. "An Accurate Pseudoempirical Model of Winding Loss Calculation in HF Foil and Round Conductors in Switchmode Magnetics", IEEE Transactions on Power Electronics, 29(8), pp. 4231-4246, 2014. https://doi.org/10.1109/TPEL.2013.2292593

[14] Orosz, T., Pánek, D., Karban, P. "FEM Based Preliminary Design Optimization in Case of Large Power Transformers", Applied Sciences, 10(4), Article number: 1361, 2020. https://doi.org/10.3390/app10041361

[15] Orosz, T., Borbély, B., Tamus, Z. Á. "Performance Comparison of Multi Design Method and Meta-Heuristic Methods for Optimal Preliminary Design of Core-Form Power Transformers", Periodica Polytechnica Electrical Engineering and Computer Science, 61(1), pp. 69-76, 2017. https://doi.org/10.3311/PPee.10207

[16] Grzesik, B., Stepien, M. "Coaxial HF Power Transformer with Tubular Linear Windings -FEM Results vs. Laboratory Test", In: 2006 12th International Power Electronics and Motion Control Conference, Portoroz, Slovenia, 2006, pp. 1313-1317. https://doi.org/10.1109/EPEPEMC.2006.4778585

[17] Boguslaw, G., Mariusz, S., Zbigniew, K., Erwin, M., Marcin, Z. "The Experimental Coaxial Transformer - Technology and Characteristics", In: 2005 European Conference on Power Electronics and Applications, Dresden, Germany, 2005, pp. P.1-P.9. https://doi.org/10.1109/EPE.2005.219379

[18] Grzesik, B., Stępień, M., Nowak, A., Smołka, J. "Influence of cooling system on the performance of coaxial transformer", presented at 13th International Symposium on Power Electronics - Ee 2005, Novi Sad, Serbia and Montenegro, Nov., 2-4, 2005.

[19] Fiolhais, M. C. N., Providência, C. "Minimum Magnetic Energy Theorem", [physics.class-ph], arXiv:0811.2598, Cornell University, Ithaca, NY, USA, 2008. [online] Available at: https://arxiv.org/ abs/0811.2598 [Accessed: 30 September 2020]

[20] Kim, D.-H., Park, J.-H. "High Efficiency Step-Down Flyback Converter Using Coaxial Cable Coupled-Inductor", Journal of Power Electronics, 13(2), pp. 214-222, 2013. https://doi.org/10.6113/JPE.2013.13.2.214

[21] Wang, R., Xiao, F., Zhao, Z., Shen, Y., Yang, G. "Effects of Asymmetric Coupling on Winding AC Resistance in MediumFrequency High-Power Transformer", IEEE Transactions on Magnetics, 50(11), pp. 1-4, 2014.

https://doi.org/10.1109/TMAG.2014.2320370
[22] Hurley, W. G., Gath, E., Breslin, J. G. "Optimizing the AC resistance of multilayer transformer windings with arbitrary current waveforms", IEEE Transactions on Power Electronics, 15(2), pp. 369-376, 2000. https://doi.org/10.1109/63.838110

[23] Pánek, D., Orosz, T., Karban, P. "Artap: Robust Design Optimization Framework for Engineering Applications", In: Third International Conference on Intelligent Computing in Data Sciences (ICDS), Marrakech, Morocco, 2019, pp. 1-6. https://doi.org/10.1109/ICDS47004.2019.8942318

[24] Karban, P., Mach, F., Kůs, P., Pánek, D., Doležel, I. "Numerical solution of coupled problems using code Agros2D", Computing, 95(special issue 1), pp. 381-408, 2013. https://doi.org/10.1007/s00607-013-0294-4

[25] Karban, P., Pánek, D., Orosz, T., Petrášová, I., Doležel, I. "FEM based robust design optimization with Agros and Ārtap", Computers \& Mathematics with Applications, 2020. https://doi.org/10.1016/j.camwa.2020.02.010

[26] Karban, P., Pánek, D., Orosz, T., Doležel, I. "Semi-analytical Solution for a Multi-objective TEAM Benchmark Problem", Periodica Polytechnica Electrical Engineering and Computer Science, in press. (Accepted for publication 18 May 2020)

[27] Grover, F. W, "Inductance Calculations: Working Formulas and Tables", Dover Publications, Mineola, NY, USA, 2004.

[28] Tóth, B., Kocsán, L. G. "Comparison of dual-mixed $h$-and $p$-version finite element models for axisymmetric problems of cylindrical shells", Finite Elements in Analysis and Design, 65, pp. 50-62, 2013. https://doi.org/10.1016/j.finel.2012.11.002

[29] Vaimann, T., Rassõlkin, A., Kallaste, A., Pomarnacki, R., Belahcen, A., Hyunh, V. K. "Artificial Intelligence in Monitoring and Diagnostics of Electrical Energy Conversion Systems", In: 2020 27th International Workshop on Electric Drives: MPEI Department of Electric Drives 90th Anniversary (IWED), Moscow, Russia, 2020, pp. 1-4. https://doi.org/10.1109/IWED48848.2020.9069566

[30] Autsou, S., Saroka, V., Karpovich, D., Rassõlkin, A., Gevorkov, L., Vaimann, T., Kallaste, A., Belahcen, A. "Comparative study of field-oriented control model in application for induction and synchronous reluctance motors for life-cycle analysis", In: 2018 25th International Workshop on Electric Drives: Optimization in Control of Electric Drives (IWED), Moscow, Russia, 2018, pp. 1-5. https://doi.org/10.1109/IWED.2018.8321371 